\title{
Butalbital Measurement
}

National Cancer Institute

\section{Source}

National Cancer Institute. Butalbital Measurement. NCI Thesaurus. Code C75365.

The determination of the amount of butalbital present in a sample. 